\title{
CDHR1-related late-onset macular dystrophy: further insights
}

Imran H. Yusuf ${ }^{1,2}$, Robert E. MacLaren ${ }^{1,2}$, Peter Charbel Issa ${ }^{1,2}$

(1) Nuffield Department of Clinical Neurosciences, University of Oxford, John Radcliffe Hospital, Oxford, OX3 9DU

(2) Oxford Eye Hospital, John Radcliffe Hospital, Oxford, OX3 9DU

Corresponding author: Professor Peter Charbel Issa. Peter.issa@ouh.nhs.uk 
We read with interest the series of patients described by Ba-Abbad and colleagues. ${ }^{1}$ Whilst biallelic truncating $C D H R 1$ variants result in cone-rod or rod-cone dystrophies consistent with the $C d h r 1 \%$ knockout mouse ${ }^{2}$ - it is intriguing that certain biallelic splice or missense variants in CDHR1 may result in late-onset macular dystrophy.

Variants in $C D H R 1$ described in association with late-onset macular dystrophy ${ }^{1}$ appear to affect the ectodomains of CDHR1. Although the 6 extracellular cadherin repeats form the longest part of the protein, they are the most conserved, suggesting an important biological function. ${ }^{3}$ Ultrastructural analysis with immunogold labelling of CDHR1 in murine retinae identified that evaginating, nascent discs at the base of the photoreceptor outer segments form CDHR1-based connections with the inner segments. ${ }^{4}$ Formed from the ectodomains of CDHR1, the contacts are lost as outer segment discs mature. This physical separation occurs through proteolytic cleavage of the CDHR1 ectodomain. ${ }^{3}$ Nontruncating $C D H R 1$ variants affecting the ectodomain may interfere with its proteolytic cleavage, or attachments to the inner segment, thereby resulting in dystrophic outer segments without widespread photoreceptor degeneration seen with biallelic truncating variants.

c.783G $>$ ACDHR1 appears to be the most common CDHR1 variant associated with late-onset macular dystrophy, based on mean allele frequency.1, 5 Indeed, when hypomorphic variants are included, CDHR1 was amongst the more common causes of macular and cone- or cone-rod dystrophy in one large series. ${ }^{6}$ Although isolated macular dystrophy appears to be the most common phenotype in c.783G $>$ ACDHR 1 homozygotes, ${ }^{5}$ peripheral retinal degeneration has been reported, ${ }^{7}$ suggesting the influence of unknown modifiers 50 which may result in rod photoreceptor degeneration instead of only macular 
involvement. The identification the inner segment binding partner of CDHR1 and the enzyme catalysing the cleavage of the CDHR1 ectodomain may help to explain the macular-predominant dystrophy in this cohort. Variants in these unknown genes may themselves act as potential disease modifiers.

55

Similar phenotypes are found in association with CDHR1, PROM1 and PRPH2 variants all of which encode proteins supporting photoreceptor outer segment structure. It remains to be determined why cones, or macular photoreceptors in general, appear to be more susceptible to the effects of specific variants in these genes.

60

Word count (350): 349

References (5): 7

65 


\section{References}

1. Ba-Abbad R, Robson AG, Mahroo OA, Wright G, Schiff E, Duignan ES, et al. A clinical study of patients with novel CDHR1 genotypes associated with late-onset macular dystrophy. Eye (London, England). 2020.

80 2. Rattner A, Smallwood PM, Williams J, Cooke C, Savchenko A, Lyubarsky A, et al. A photoreceptor-specific cadherin is essential for the structural integrity of the outer segment and for photoreceptor survival. Neuron. 2001;32(5):775-86.

3. Rattner A, Chen J, Nathans J. Proteolytic shedding of the extracellular domain of photoreceptor cadherin. Implications for outer segment assembly. The Journal of biological chemistry. 2004;279(40):42202-10.

4. Burgoyne T, Meschede IP, Burden JJ, Bailly M, Seabra MC, Futter CE. Rod disc renewal occurs by evagination of the ciliary plasma membrane that makes cadherinbased contacts with the inner segment. Proc Natl Acad Sci U S A. 2015;112(52):15922-7. 5. Charbel Issa P, Gliem M, Yusuf IH, Birtel J, Müller PL, Mangold E, et al. A Specific Macula-Predominant Retinal Phenotype Is Associated With the CDHR1 Variant c.783G>A, a Silent Mutation Leading to In-Frame Exon Skipping. Invest Ophthalmol Vis Sci. 2019;60(10):3388-97.

6. Birtel J, Eisenberger T, Gliem M, Muller PL, Herrmann P, Betz C, et al. Clinical and genetic characteristics of 251 consecutive patients with macular and cone/cone-rod dystrophy. Sci Rep. 2018;8(1):4824.

7. Bessette AP, DeBenedictis MJ, Traboulsi EI. Clinical characteristics of recessive retinal degeneration due to mutations in the CDHR1 gene and a review of the literature. Ophthalmic Genet. 2018;39(1):51-5. 